\title{
Conjunctival Metastasis from Pleural Mesothelioma
}

\author{
Andrew Chung ${ }^{\text {a }}$ Hardeep Singh Mudhar ${ }^{b}$ \\ a Department of Ophthalmology, Pinderfields Hospital, Mid-Yorkshire Hospitals NHS Trust, \\ Wakefield, and ${ }^{\mathrm{b}}$ National Specialist Ophthalmic Pathology Service (NSOPS), Department of \\ Histopathology, Royal Hallamshire Hospital, Sheffield, UK
}

\section{Key Words}

Conjunctival metastasis · Pleural mesothelioma - Metastatic mesothelioma

\begin{abstract}
Purpose of the Study: To describe the ophthalmic and pathological features of a conjunctival tumour in a 76-year-old male with terminal pleural mesothelioma who developed a rapidly growing mass on the bulbar conjunctiva. Procedures: The patient presented with a rapidly growing, pink nodule on the right nasal bulbar conjunctiva. His past medical history included biopsy-confirmed pleural malignant mesothelioma treated unsuccessfully with chemoradiotherapy. By the time he presented with his ocular signs, the systemic management plan was palliative. An incisional biopsy of the conjunctival lesion was performed, stained with haematoxylin and eosin and exposed to immunohistochemistry. Results: The histology showed a solid and pseudo-papillary tumour. The tumour was positive for cytokeratin (AE1/AE3), calretinin, WT1 and mesothelin, confirming metastatic mesothelioma to the conjunctiva. Conclusions and Message of the Paper: The clinical features mimicked metastatic carcinoma, and furthermore, the tumour's immunohistochemical positivity for cytokeratin could have led to an erroneous diagnosis of carcinoma rather than mesothelioma. The two key messages are (1) that metastatic mesothelioma can mimic metastatic carcinoma clinically and (2) that any cytokeratin-positive, rapidly growing conjunctival tumour with unusual morphology should be stained immunohistochemically with a mesothelioma panel to exclude metastatic mesothelioma.
\end{abstract}

(c) 2014 S. Karger AG, Basel

\section{Introduction}

Metastatic tumours to the conjunctiva are a rare event and often reflect an advanced state of disseminated malignancy. In one large series of 2,455 conjunctival lesions that were biopsied, 1 was a metastasis [1]. In another large series reviewing 1,643 melanocytic and non- 
Ocular Oncology and Pathology

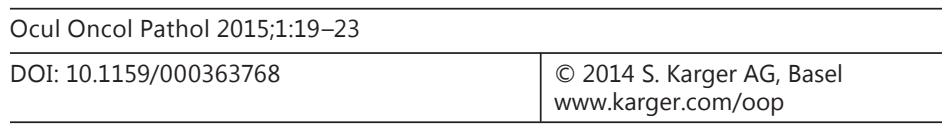

Chung and Mudhar: Conjunctival Metastasis from Pleural Mesothelioma
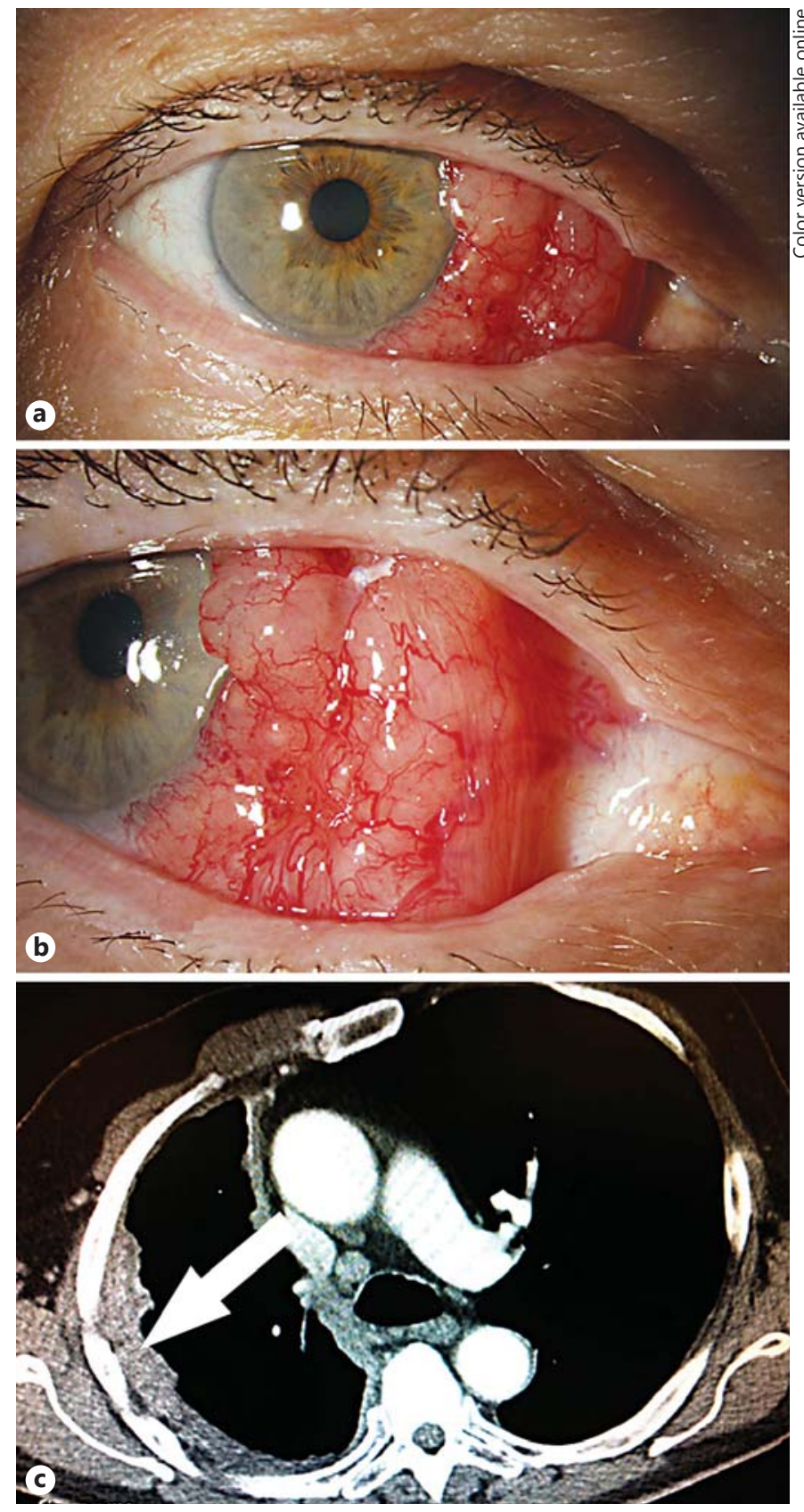

Fig. 1. a Right eye showing a bulbar conjunctival mass. b Note the multinodular nature and vascularity at the higher magnification. c Thoracic CT scan showing pleural mesothelioma on the right side (arrow).

melanocytic conjunctival tumours, only 13 cases represented metastatic tumours to the conjunctiva $(<1 \%)$ [2]. In a further study of 10 conjunctival metastases, the tumour originated from the breast ( 4 cases), lung ( 2 cases), cutaneous melanoma ( 2 cases) or larynx ( 1 case) or had an undetermined origin (4 cases) [3]. Conjunctival metastases tend to present as rapidly growing, yellow or fleshy nodules and can be nodular or sessile, multifocal or diffuse [3]. In this case report, we describe the clinical and histopathological features of a rapidly growing, fleshy conjunctival tumour in a 76-year-old man with terminal pleural mesothelioma. 


\section{Ocular Oncology} and Pathology

Fig. 2. a-d Primary pleural mesothelioma. a Solid area of discohesive epithelioid cells. Inset Pseudo-papillary area of the same tumour. H\&E. $\times 200 . \quad$ b Pan-cytokeratin immunopositivity. $\times 400$. c Nuclear calretinin immunopositivity. $\times 400$. d Nuclear WT1 immunopositivity. $\times 400$. eh Metastatic conjunctival mesothelioma. e Discohesive epithelioid cells. Inset Pseudo-papillary area of the same tumour. H\&E. $\times 400$. f Pan-cytokeratin immunopositivity. $\times 400$. g Nuclear calretinin immunopositivity. $\times 400$. $\mathbf{h}$ Nuclear WT1 immunopositivity. $\times 400$.

\begin{tabular}{l|l}
\hline Ocul Oncol Pathol 2015;1:19-23 \\
\hline DOI: 10.1159/000363768 & $\begin{array}{l}\text { @ 2014 S. Karger AG, Basel } \\
\text { www.karger.com/oop }\end{array}$ \\
\hline
\end{tabular}

Chung and Mudhar: Conjunctival Metastasis from Pleural Mesothelioma

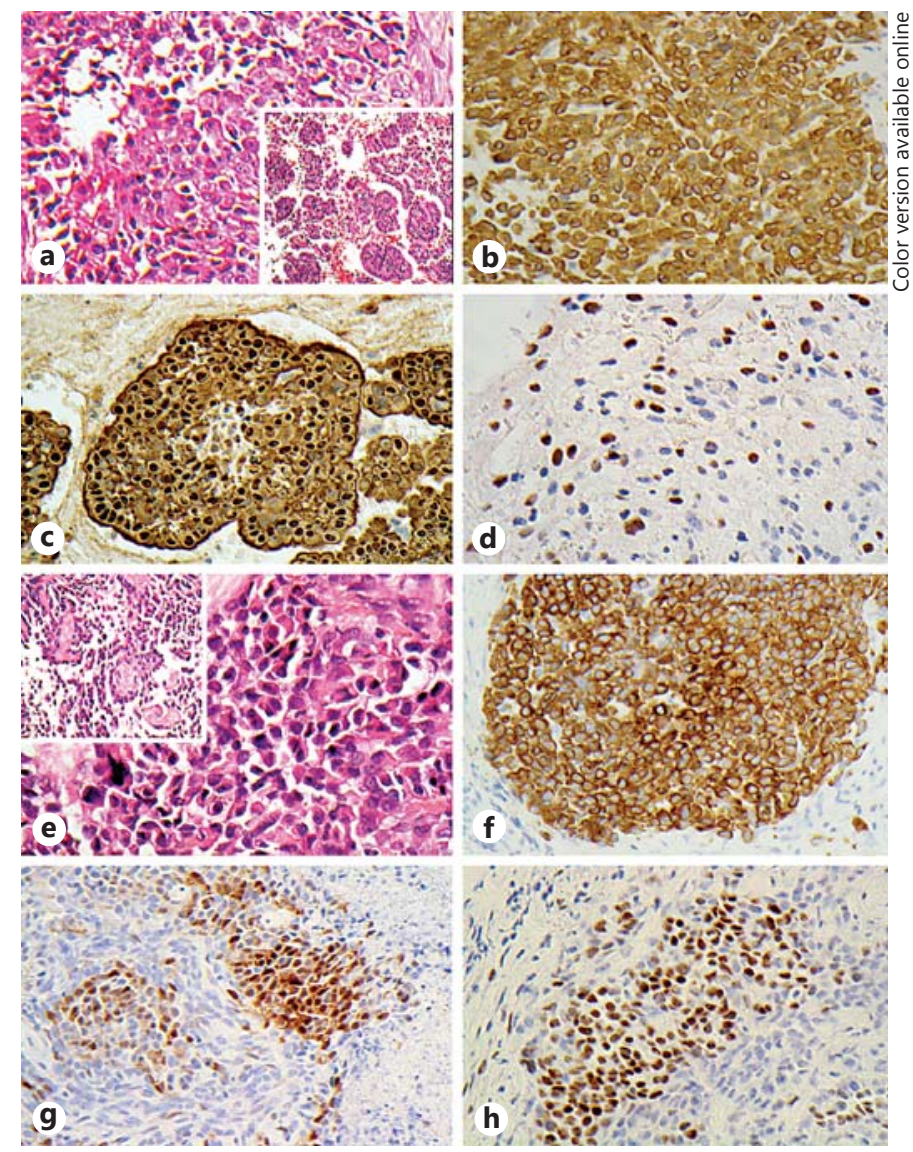

\section{Case Report}

A 76-year-old male presented with a rapidly expanding, solitary lump on the nasal bulbar conjunctiva of the right eye which he had noticed 4 weeks previously (fig. 1a, b) and which was associated with dull discomfort. There was no previous ophthalmic history. There was no evidence of bleeding or discharge. He was a retired coal miner and had been diagnosed with biopsy-proven right pleural mesothelioma 2 years prior to the onset of his eye symptoms (fig. 1c, fig. $2 a-d$ ), for which he had undergone multimodality radiotherapy and chemotherapy. He had failed to respond to treatment, and by the time he was referred to the ophthalmology department for his conjunctival lesion, the objective of his overall treatment was palliative.

On examination, the best-corrected visual acuities on the logMAR chart were 0.14 (right eye) and 0.16 (left eye). There was a solid, pink, fleshy, multinodular, non-mobile, vascularised conjunctival mass on the nasal bulbar conjunctiva, extending from the corneoscleral limbus to the equator (fig. 1a, b). It measured 18 $\mathrm{mm}$ in maximum dimension across the base. The rest of the ophthalmic examination was unremarkable. A CT scan showed no evidence of orbital disease. An incisional biopsy was taken from the conjunctival lesion. The biopsy tissue was fixed in $10 \%$ buffered formalin and processed to paraffin sections; 4 - $\mu$ m sections were cut, mounted on glass slides and stained with conventional haematoxylin and eosin (H\&E). Some sections were exposed to the immunohistochemistry panel outlined below.

The histology of the incisional biopsy showed a solid and pseudo-papillary lesion composed of epithelioid cells with eosinophilic cytoplasm and focal tumour necrosis (fig. 2e). The histological differential diagnosis included metastatic carcinoma, mesothelioma and melanoma. A detailed immunohistochemistry panel was carried out and showed the following profile: the tumour was positive for AE1/AE3 (pan-cytokeratin; fig. 2f), calretinin (fig. 2g) and WT1 (fig. 2h) as well as focally positive for mesothelin (not shown). The tumour was negative for BerEp4, TTF-1, S100, melan-A and claudin-4 (not shown). The negative panel excluded melanoma (negative for melan-A and S100) and metastatic carcinoma of the lung (negative for BerEp4, TTF-1 and claudin-4). A review of the previous thoracic biopsy showed an identical solid and pseudo- 
papillary, partly necrotic tumour composed of epithelioid cells, exhibiting an immunohistochemical profile identical to that of the conjunctival metastasis. The conjunctival tumour therefore represented a metastatic pleural mesothelioma.

The patient was referred for the opinion of a specialist in ocular oncology. It was felt that the prognosis was extremely poor in view of the terminal nature of the mesothelioma and that it was inappropriate to use highly invasive treatment with significant potential adverse effects but that local radiotherapy may help. However, the patient died of pleural mesothelioma before ocular radiotherapy could be commenced.

\section{Discussion}

Metastatic carcinomas to the conjunctiva present with rapidly growing, fleshy, pink, vascularised masses [3], which is identical to the clinical presentation of the metastatic mesothelioma in this case. In the absence of a previous history of mesothelioma, the lesion could quite easily have been mistaken for a metastatic carcinoma, not only from a clinical perspective but also from a histological perspective, given the strong immunohistochemical expression of cytokeratins in both tumours. The clinical differential diagnosis would also include primary carcinomas such as squamous or sebaceous carcinomas, lymphoma or amelanotic melanoma, but the previous history and access to the previous histological pleural biopsy material permitted a secure histopathological diagnosis.

Pleural mesothelioma has a dire prognosis, with $75 \%$ of patients dead within a year of diagnosis [4]. Around $80 \%$ of patients with pleural mesothelioma report previous occupational exposure to asbestos, with a latency period from exposure to tumour development of up to 20 years [5]. The main carcinogenic agents are crocidolite and amosite asbestos fibres, and those occupations that are classically associated with asbestos exposure include mining, ship building and insulation work, to name but a few [6]. The incidence is $0.1-0.2$ per 100,000 population in males and females, with the male-to-female ratio being 3:1 [7]. Most cases develop in the 5th to 7th decade of life [4]. Pleural mesothelioma is staged using MRI scanning and laparoscopic thoracoscopy according to the TNM classification [8]. Treatment modalities include surgery, chemotherapy, radiation or a combination of these. Without treatment, around $75 \%$ of patients die within a year of diagnosis. With combination treatment, some publications quote a survival period of 16-19 months, with a small number of patients surviving longer $[9,10]$.

Our patient had been a coal miner. This is not a classic asbestos exposure occupation, so the presumed aetiology was either incidental environmental exposure or idiopathic [11]. Previous autopsy studies have documented metastases in 50\% of cases of both pleural and peritoneal mesothelioma, with common sites being the lymph nodes, lung, liver, pleura, bone, adrenal glands and heart [4]. In this case, the conjunctiva was the only extrathoracic site for metastasis. This is similar to a previous case of metastatic pleural mesothelioma to the left upper eyelid (without conjunctival involvement) that presented with recurrent chalazia, with multiple nodules under the skin of the eyelid showing externally [12]. Two previous reports have also highlighted orbital metastases from pleural and peritoneal mesothelioma presenting with proptosis in the absence of conjunctival involvement $[13,14]$. All these cases of ocular metastatic mesothelioma were diagnosed in known cases of histologically confirmed pleural or peritoneal mesothelioma.

The histological morphology alone is not sufficient to make a reliable diagnosis of malignant mesothelioma (especially the epithelioid variant) because of its similarity to pulmonary carcinomas (especially adenocarcinomas). Immunohistochemistry utilising a panel of antibodies must be applied to allow a distinction from pulmonary adenocarcinoma - a critical decision, as a diagnosis of mesothelioma entails a very poor prognosis, a poor 
response to therapy and, crucially, industrial compensation. This panel usually comprises antibodies that preferentially stain pulmonary adenocarcinoma (such as TTF-1, BerEp4 and claudin-4) and those that preferentially stain mesothelioma (calretinin, CK5/6, WT1 and D240). For calretinin, a meta-analysis reported a sensitivity and a specificity of 82 and $85 \%$, respectively, for epithelioid mesothelioma [15]. For WT1, the same study reported a sensitivity and a specificity of 77 and 96\%, respectively, for epithelioid mesothelioma [15]. Other tumours stained with calretinin include ovarian sex cord stromal tumour, cardiac myxoma and a small number of poorly differentiated malignancies, whereas WT1 stains a wider variety of non-mesothelioma malignancies. Calretinin therefore possesses positivity in a small, select number of lesions, and we recommend its use in cases of histologically atypically appearing conjunctival lesions, where a metastasis is being considered.

In summary, this case report for the first time describes the clinical and immunohistochemical findings of malignant pleural mesothelioma metastatic to the conjunctiva. Furthermore, we recommend that any tumour suspected to be metastatic to the conjunctiva, and considered to be a carcinoma on the basis of histology and cytokeratin positivity, be immunohistochemically stained with at least calretinin and WT1 to exclude metastatic mesothelioma.

\section{Disclosure Statement}

The authors declare that there are no conflicts of interest. Also, there are no financial interests.

\section{References}

$>1$ Grossniklaus HE, Green WR, Luckenbach M, Chan CC: Conjunctival lesions in adults: a clinical and histopathologic review. Cornea 1987;6:78-116.

$\checkmark 2$ Shields CL, Demirci H, Karatza E, Shields JA: Clinical survey of 1,643 melanocytic and nonmelanocytic conjunctival tumors. Ophthalmology 2004;111:1747-1754.

-3 Kiratli H, Shields CL, Shields JA, DePotter P: Metastatic tumours to the conjunctiva: report of 10 cases. Br J Ophthalmol 1996;80:5-8.

4 Churg A: Neoplastic asbestos-induced disease; in Churg A, Green FHY (eds): Pathology of Occupational Lung Disease, ed 2. Baltimore, Williams \& Wilkins, 1998, p 339.

$\checkmark 5$ Hodgson JT, Darnton A: The quantitative risks of mesothelioma and lung cancer in relation to asbestos exposure. Ann Occup Hyg 2000;44:565-601.

6 Yang H, Testa JR, Carbone M: Mesothelioma epidemiology, carcinogenesis, and pathogenesis. Curr Treat Options Oncol 2008;9:147-157.

7 Ascoli V, Scalzo CC, Facciolo F, et al: Malignant mesothelioma in Rome, Italy 1980-1995: a retrospective study of 79 patients. Tumori 1996;82:526-532.

$>8$ Rusch VW: A proposed new international TNM staging system for malignant pleural mesothelioma. From the International Mesothelioma Interest Group. Chest 1995;108:1122-1128.

-9 Sugarbaker DJ, Garcia JP, Richards WG, et al: Extrapleural pneumonectomy in the multimodality therapy of malignant pleural mesothelioma: results in 120 consecutive patients. Ann Surg 1996;224:288-294.

10 Huncharek M, Kelsey K, Mark EJ, et al: Treatment and survival in diffuse malignant pleural mesothelioma: a study of 83 cases from the Massachusetts General Hospital. Anticancer Res 1996;16:1265-1268.

11 Wei B, Jia X, Ye B, Yu J, Zhang B, Zhang X, et al: Impacts of land use on spatial distribution of mortality rates of cancers caused by naturally occurring asbestos. J Expo Sci Environ Epidemiol 2012;22:516-521.

12 Tsina EK, Papaioannou D, Matsouka FO, Kosmidis PA: Metastatic pleural mesothelioma initially masquerading as chalazion. Br J Ophthalmol 2006;90:921-922.

13 Godwin MC, Penn RF: Diffuse peritoneal mesothelioma with metastasis to the orbital area as a presenting symptom. Am J Ophthalmol 1957;43:213-219.

14 Feinmesser M, Hurwitz JJ, Heathcote JG: Pleural malignant mesothelioma metastatic to the orbit. Can J Ophthalmol 1994;29:193-197.

15 King JE, Thatcher N, Pickering CA, Hasleton PS: Sensitivity and specificity of immunohistochemical markers used in the diagnosis of epithelioid mesothelioma: a detailed systematic analysis using published data. Histopathology 2006;48:223-232. 